\title{
COMPLEX ROOTS OF REAL CHARACTERISTIC FUNCTIONS
}

\author{
ROBERT G. STAUDTE, JR. ${ }^{1}$ AND MAHABANOO N. TATA \\ Abstract. For each positive rational $r$ which is not the recipro- \\ cal of an integer a complex-valued characteristic function is con- \\ structed whose $r$ th power is a real-valued characteristic function.
}

1.1. Introduction. We shall call a distribution function $F$ "symmetric" if $F(x)+F(-x-0)=1$ for all $x$. It is known that if $F$ is symmetric, then the convolution $F * F$ is also symmetric. In this paper we examine the converse proposition:

If $F * F$ is symmetric, then $F$ is symmetric.

In $\$ 2.1$ we construct examples which show that (1.1) cannot be proved without additional assumptions. In order that (1.1) be true it is sufficient that the characteristic function of $F$ be infinitely divisible or analytic; this is discussed further in $\$ 4.1$.

In $\$ 2.2$ we generalize and show that for $n=2,3, \cdots$ there exists a complex-valued characteristic function $\phi_{n}$ such that for positive integers $m, \phi_{n}^{m}$ is real-valued if and only if $n$ divides $m$. Furthermore for each positive rational $r$ which is not the reciprocal of an integer there exists a complex-valued characteristic function $\phi_{r}$ such that $\phi_{r}^{r}$ is a real-valued characteristic function.

We shall make use of the following theorem several times in this paper.

THEOREM 1.1 ([1, P. 73]). Let $\phi$ be a real-valued function which satisfies the following conditions for a fixed positive real $r$ :

(i) $\phi(0)=1$ and $\phi(r)=0$.

(ii) $\phi(t) \geqq 0$ for $t$ in $(-r, r)$.

(iii) $\phi$ is convex and continuous in the interval $[0, r]$.

(iv) $\phi(t)=\phi(-t)$ for $t$ in $[-r, r]$.

(v) $\phi$ is periodic with period $2 r$.

Then $\phi$ is the characteristic function of a lattice distribution.

Presented to the Society, January 23, 1970; received by the editors October 3, 1969.

AMS Subject Classifications. Primary 6020; Secondary 4255.

Key Words and Phrases. Convolutions, distribution functions, roots of characteristic functions, symmetric distribution functions.

${ }^{1}$ Research supported by NSF Grant GP-13484 
(1.2) We note that if a real-valued function $\phi$ defined on $[0, r]$ satisfies conditions (i)-(iii) it may be uniquely extended to a realvalued periodic characteristic function $\tilde{\phi}$ with period $2 r$. No confusion should result if we drop the tilde on $\tilde{\phi}$.

When convenient, we shall express $\phi(t)$ as $u(t)+i v(t)$ or $\rho(t) e^{i \theta(t)}$, where $u(t)=\operatorname{Re} \phi(t), v(t)=\operatorname{Im} \phi(t), \rho(t)=|\phi(t)|$ and $\theta(t)=\operatorname{Arg} \phi(t)$, $-\pi<\theta(t) \leqq \pi$. These symbols are of ten used as generics within a class; they may be subscripted to distinguish between classes.

\subsection{Complex square roots of real characteristic functions.}

THEOREM 2.1. There exists a family of asymmetric lattice distributions $F$ such that $F * F$ is symmetric.

It is clearly sufficient to construct a class of periodic characteristic functions $\phi$ such that $\phi$ is complex-valued, but $\phi^{2}$ is real-valued. We shall carry out this construction in the following lemmas.

Lemma 2.1. Let $A=\{t: \cos t \geqq 0\}$. Given an arbitrary characteristic function $\phi_{1}=u_{1}+i v_{1}$ and any real-valued characteristic function $\phi_{2}$ which satisfies conditions (i) $-(\mathrm{v})$ of Theorem 1.1 for $r=\pi / 2$ (the $\pi$-periodic function defined by $\phi_{2}(t)=1-\sin |t|,|t| \leqq \pi / 2$, would do); then the function $\phi$ defined by

$$
\begin{aligned}
\phi(t) & =u_{1}(t) \phi_{2}(t), & & t \in A, \\
& =\frac{u_{1}(t) \phi_{2}(t)}{2}+i \frac{v_{1}(t) \phi_{2}(t)}{2}, & & t \in \bar{A}
\end{aligned}
$$

is a characteristic function.

Proof. Let $\phi_{3}$ be defined on $[0, \pi]$ by $\phi_{3}(t)=\phi_{2}(t)$ for $0 \leqq t \leqq \pi / 2$ and $\phi_{3}(t)=0$ for $\pi / 2<t \leqq \pi$. By remark (1.2), $\phi_{3}$ may be uniquely extended to a real-valued characteristic function $\phi_{3}$ with period $2 \pi$; then $\phi_{3}=\phi_{2}$ on $A$ and $\phi_{3}=0$ on $\bar{A}$.

Now

$$
\phi=\left(\overline{\phi_{1} \phi_{3}}+\phi_{1} \phi_{2}\right) / 2
$$

is a characteristic function which is real-valued on $A$. It is clear that $\phi$ may be written in the form (2.1).

LEMмA 2.2. There exists a characteristic function $\phi_{7}=u_{7}+i v_{7}$ with $u_{7}=0$ on $\bar{A}$ and $v_{7}(t) \neq 0$ on $\bar{A}$ except when $t$ is an integral multiple of $\pi$.

Proof. Let $\phi_{4}$ be a real-valued characteristic function of period $\pi$ which satisfies conditions (i)-(v) of Theorem 1.1 and also 
(vi) $\phi_{4}(t)$ is positive for $t$ in $(0, \pi / 2)$.

(vii) $\phi_{4}(t) \cos t$ is convex for $t$ in $(0, \pi / 2)$. (An example is the $\pi$-periodic real-valued extension (see remark (1.2)) of $\phi_{4}(t)=e^{-t}(1-\sin t), t$ in $[0, \pi / 2]$.)

Define $\phi_{5}$ on $[0, \pi / 2]$ by $\phi_{5}(t)=\phi_{4}(t) \cos t$. By remark (1.2), $\phi_{5}$ may be extended to a real-valued characteristic function $\phi_{5}$ of period $\pi$. Note that $\phi_{5}(t)=\phi_{4}(t)|\cos t|$ for all $t$.

Now consider the characteristic function $\phi_{6}$ defined by $\phi_{6}(t)=e^{i t} \phi_{4}(t)$ for all $t$. Clearly $u_{6}=\phi_{5}$ on $A$ and $u_{6}=-\phi_{5}$ on $\bar{A}$. In addition, $v_{6}(t)=\phi_{4}(t) \sin t \neq 0$ except when $t$ is an integral multiple of $\pi / 2$.

Finally, define the characteristic function $\phi_{7}$ by $\phi_{7}=\left(\phi_{5}+\phi_{6}\right) / 2$. Then $u_{7}=\phi_{5}$ on $A$ and $u_{7}=0$ on $\bar{A}$, while $v_{7}(t)=\left(\phi_{4}(t) \sin t\right) / 2 \neq 0$ on $\bar{A}$, except when $t$ is an integral multiple of $\pi$.

Proof of Theorem 2.1. We substitute $\phi_{7}$ of Lemma 2.2 for the $\phi_{1}$ of Lemma 2.1 to obtain a characteristic function $\phi$ of the form

$$
\begin{aligned}
\phi(t) & =\phi_{2}(t) \phi_{4}(t) \cos t, & & t \in A, \\
& =i\left(\frac{\phi_{2}(t) \phi_{4}(t) \sin t}{4}\right), & & t \in \bar{A} .
\end{aligned}
$$

It is clear that $\phi$ is complex-valued, but $\phi^{2}$ is real-valued. Since $\phi_{2}$ and $\phi_{4}$ are both periodic, so is $\phi$; moreover $t_{0}=2 \pi$ is the least positive value for which $\left|\phi\left(t_{0}\right)\right|=1$. Hence $\phi$ has period $2 \pi$ and is the characteristic function of a lattice distribution [1, p. 25].

COROLlARY 2.1. There exist absolutely continuous and asymmetric distributions $F$ such that $F * F$ is symmetric. by

Proof. Consider the Pólya-type characteristic function $\psi$ defined

$$
\begin{aligned}
\psi(t) & =1-\sin \frac{|t|}{2}, & & |t| \leqq \pi, \\
& =0, & & |t|>\pi .
\end{aligned}
$$

$\psi$ is the characteristic function of an absolutely continuous distribution $[1$, p. 70$]$, so $\phi \psi$ is the characteristic function of an absolutely continuous distribution. It is clear that $\phi \psi$ satisfies the requirements of the corollary.

Corollary 2.2. A real-valued characteristic function may have two distinct "square roots," each of which is a characteristic function.

Proof. Let $\phi$ be the characteristic function constructed in Theorem 2.1. Then $\phi \neq \bar{\phi}$, but $\phi^{2}=\bar{\phi}^{2}$. 
2.2. Complexvalued $r$ th roots of real characteristic functions. In this section we use the examples constructed in $\$ 2.1$ to show (Theorem 2.2) that for each integer $n>1$, there exists an asymmetric discrete distribution function $F_{n}$ whose $m$-fold convolution is symmetric if and only if $n$ divides $m$.

THEOREM 2.2. For each integer $n>1$, there exists a periodic complexvalued characteristic function $\phi_{n}$ such that for each positive integer $m$, $\phi_{n}^{m}$ is real-valued if and only if $n$ divides $m$.

Proof. We may choose $\phi_{2}$ and $\phi_{4}$ in the proof of Theorem 2.1 so that the functions $\phi_{8, K}, 0<K<1$, defined by (2.3) and (2.4) below satisfy conditions (i)-(iii) of Theorem 1.1.

$$
\begin{array}{ll}
\phi_{8, K}(t)=K \phi_{2}(t) \phi_{4}(t) \sin t / 4, & t \in[\pi / 4, \pi / 2], \\
\phi_{8, K}(t)=(4 / \pi)\left(\phi_{8, K}(\pi / 4)-1\right) t+1, & t \in[0, \pi / 4) .
\end{array}
$$

(For example, let $\phi_{2}(t)=1-\sin t, \phi_{4}(t)=e^{-t}(1-\sin t)$ for $t$ in $[0, \pi / 2]$.) By remark (1.2) we may extend $\phi_{8, K}$ to a real-valued $\pi$-period characteristic function which we also call $\phi_{8, K}, 0<K<1$.

Now let $\phi$ be the characteristic function of Theorem 2.1 that is determined by the above choice of $\phi_{2}$ and $\phi_{4}$, and define the class of characteristic functions $\phi_{9, K}=u_{9, K}+i v_{9, K}, \quad 0<K<1$, by $\phi_{9, K}$ $=\left(\phi_{8, K} \phi+\phi^{2}\right) / 2$. The definition of $\phi_{9, K}$ and (2.2) together imply that

$$
\begin{aligned}
u_{9, K}(t) & =\left(\phi_{8, K}(t) u(t)+u^{2}(t)\right) / 2, & & t \in A, \\
& =-v^{2}(t) / 2, & & t \in \bar{A} ; \\
v_{9, K}(t) & =0, & & \\
& =\phi_{8, K}(t) v(t) / 2, & t \in A, \bar{A} . &
\end{aligned}
$$

Let $B=\{t: \cos 2 t \geqq 0\}$. By construction-see (2.2), (2.3), and (2.4) -we have

$$
\phi_{8, K}(t)=+K v(t) \quad \text { or } \quad-K v(t) \quad \text { for each } t \text { in } \bar{A} \cap \bar{B} .
$$

In Lemma 2.1 replace $r=\pi / 2$ by $r=\pi / 4, A$ by $B, \phi_{1}$ by $\phi_{9, K}$, and $\phi_{2}$ by $\phi_{10}$, where $\phi_{10}$ is a real-valued $\pi / 2$-periodic characteristic function which satisfies conditions (i) $-(v)$ of Theorem 1.1. Then we obtain from (2.1) a family of characteristic functions $\phi_{11, K}, 0<K<1$, with

$$
\begin{aligned}
\phi_{11, K}(t) & =u_{9, K}(t) \phi_{10}(t), & & t \in B, \\
& =u_{9, K}(t) \phi_{10}(t) / 2+i v_{9, K}(t) \phi_{10}(t) / 2, & & t \in \bar{B}
\end{aligned}
$$

which together with (2.5) and (2.6) yields 


$$
\begin{array}{rlrl}
u_{11, K}(t) & =\phi_{10}(t)\left[\phi_{8, K}(t) u(t)+u^{2}(t)\right] / 2, & & t \in A \cap B, \\
& =\phi_{10}(t)\left[\phi_{8, K}(t) u(t)+u^{2}(t)\right] / 4, & & t \in A \cap \bar{B}, \\
& =-\phi_{10}(t) v^{2}(t) / 2, & & t \in \bar{A} \cap B, \\
& =-\phi_{10}(t) v^{2}(t) / 4, & & t \in \bar{A} \cap \bar{B}, \\
v_{11, K}(t) & =0, & t \in A \cup B, \\
& =\phi_{10}(t) \phi_{8, K}(t) v(t) / 4, & t \in \bar{A} \cap \bar{B} .
\end{array}
$$

Now each $v_{11, K}$ is nonzero on a nonempty subset of $\bar{A} \cap \bar{B}$, which without loss of generality we take to be $\bar{A} \cap \bar{B}$ itself. Let $C=\left\{t: v_{11, K}(t)>0\right\}$; then $C$ does not depend on $K$ and $\bar{A} \cap \bar{B}$ $=\{t:|t| \in C\}$. Moreover, by (2.7), (2.8), and (2.9), we have $\theta_{11, K}(t)$ $=\operatorname{Arg} \phi_{11, K}(t)=\operatorname{Tan}^{-1} K$ for $t$ in $C$ and $-\operatorname{Tan}^{-1} K$ for $-t$ in $C$. The only other values of $\theta_{11, K}$ are 0 and $\pi$.

Finally we define the class of characteristic functions $\Phi$ by $\Phi=\left\{\phi_{11, K}^{4}: 0<K<1\right\}$. Then every member of $\Phi$ has a uniquely defined argument of the form

$$
\begin{array}{rlrl}
\theta(t) & =0, & t & \in A \cup B, \\
& =\pi / \alpha, & t \in C, \\
& =-\pi / \alpha, & -t \in C,
\end{array}
$$

where $\alpha$ is determined by $\pi / \alpha=4 \operatorname{Tan}^{-1} K, 0<K<1$. We shall index $\Phi$ by $\alpha$ in $(1, \infty)$. For $\beta>0$ we now define $\phi^{\beta}$ by $\phi^{\beta}(t)=\rho^{\beta}(t) e^{i \beta \theta(t)}$; then it is clear that each $\phi_{\alpha} \in \Phi$ satisfies the condition

(2.11) $\phi_{\alpha}^{\beta}$ is a well-defined function for $\beta \geqq 0$ which is real-valued if and only if $\beta$ is an integral multiple of $\alpha$.

It is not clear that $\phi \in \Phi$ implies $\phi^{\beta}$ is a characteristic function except for the case of integer valued $\beta>1$. (But see Corollary 2.5.) The proof of Theorem 2.2 is complete upon observation that each $\phi_{11, K}$, $0<K<1$, hence each $\phi_{\alpha} \in \Phi$, is periodic.

Corollary 2.3. For each integer $n>1$ there exists absolutely continuous and asymmetric distributions $F_{n}$ such that the $m$-fold convolution of $F_{n}$ is asymmetric for $m<n$ but symmetric for $m=n$.

Proof. The proof is similar to that of Corollary 2.1 and will therefore be omitted.

COROLlary 2.4. For each integer $n>1$ there exists a real-valued characteristic function $\psi_{n}$ which has distinct complex-valued jth roots (which are characteristic functions) for $j=2,3, \cdots, n$. 
Proof. Given $n$, we choose $\phi_{\alpha} \in \Phi$, where $\alpha=n$ !. Then by (2.11), $\phi_{n !}^{\beta}$ is complex-valued for $0<\beta<n$ ! and $\psi_{n} \equiv \phi_{n !}^{n !}$ is a real-valued characteristic function. Hence for each $j=2,3, \cdots, n, \phi_{n !}^{n ! / j}$ and $\bar{\phi}_{n !}^{n ! / j}$ are distinct complex-valued characteristic functions whose $j$ th powers equal $\psi_{n}$.

COROLlaRy 2.5. For each rational $r>0$ which is not the reciprocal of an integer, there exists a complex-valued characteristic function $\psi_{r}$ such that $\psi_{r}^{r}$ is a real-valued characteristic function.

If $r$ is the reciprocal of an integer, no such $\psi_{r}$ exists.

Proof. Let $r=m / n$, where $m, n>1$ are relatively prime integers. Choose $\phi_{m}, \phi_{n} \in \Phi$ and define $\psi_{r}=\left(\phi_{m} \phi_{n}\right)^{n^{2}}$. Since $m$ does not divide $n^{2}, \psi_{r}$ is a complex-valued characteristic function. Moreover, $\psi_{r}^{r}=\left(\phi_{n}^{m n}\right)\left(\phi_{m}^{m n}\right)$ is a real-valued characteristic function.

If $r=1 / n, n$ a positive integer, and $\psi_{r}^{r}$ is real-valued, then $\psi_{r}$ must also be real-valued since $\psi_{r}=\left(\psi_{r}^{r}\right)^{n}$.

REMARK. It may be possible to extend this corollary to the case of irrational $r>0$, but this extension is not obvious.

\subsection{An asymmetric probability distribution which has a symmetric} convolution. The examples in $\$ \$ 2.1$ and 2.2 do not contain easily inverted characteristic functions. Below we give an example of a complex-valued characteristic function whose square is real-valued and its associated (lattice) distribution.

Proposition 3.1. Let $\phi$ be periodic with period $2 \pi$ and let it be defined on $[-\pi, \pi]$ as follows.

$$
\begin{aligned}
\phi(t) & =i m\{|t+3 \pi / 4|-\pi / 4\}, & & -\pi<t \leqq-\pi / 2, \\
& =\left(4 / \pi^{2}\right)(t+\pi / 2)^{2}, & & -\pi / 2<t \leqq 0, \\
& =\left(4 / \pi^{2}\right)(t-\pi / 2)^{2}, & & 0<t \leqq \pi / 2, \\
& =-i m\{|t-3 \pi / 4|-\pi / 4\}, & & \pi / 2<t \leqq \pi,
\end{aligned}
$$

where

$$
i^{2}+1=0 \text { and } m>0 .
$$

Then for sufficiently small $m$ (say $\left.m<1 / \pi^{2}\right) \phi$ is a characteristic function. It is clear that $\phi^{2}$ is real-valued.

Proof. We find the Fourier coefficients for $\phi$.

Writing

$$
\phi(t)=\sum_{0}^{\infty} a_{k} \cos k t+i \sum_{0}^{\infty} b_{k} \sin k t
$$


we have

$$
\begin{aligned}
\pi a_{0} & =\pi / 6 \\
\frac{\pi}{2} a_{k} & =\frac{2}{k^{2}}\left\{\frac{2}{\pi}-\frac{4}{k \pi^{2}} \sin k \frac{\pi}{2}\right\}, \\
\frac{\pi}{2} b_{k} & =\frac{m}{k^{2}}\left\{2 \sin \frac{3 \pi k}{4}-\sin \frac{k \pi}{2}\right\} .
\end{aligned}
$$

We shall now construct a probability function on the integers with characteristic function $\phi$ as above.

Let $X$ be a random variable with

$$
\begin{aligned}
P\{X=k\} & =r_{k}, & & k=-1,-2, \cdots, \\
& =p_{0}, & k & =0, \\
& =p_{k}, & k & =1,2, \cdots .
\end{aligned}
$$

Then the characteristic function of $X$ is defined by

$$
\tilde{\phi}(t)=p_{0}+\sum_{k=1}^{\infty}\left(p_{k}+r_{-k}\right) \cos k t+\sum_{k=1}^{\infty}\left(p_{k}-r_{-k}\right) \sin ^{n} k t .
$$

We equate the Fourier coefficients of $\phi$ and $\tilde{\phi}$ to obtain

$$
p_{0}=1 / 6 \text {, }
$$

(3.2) $\frac{\pi}{2}\left(p_{k}+r_{-k}\right)=\frac{2}{k^{2}}\left\{\frac{2}{\pi}-\frac{4}{k \pi^{2}} \sin \frac{k \pi}{2}\right\}$,

$$
\frac{\pi}{2}\left(p_{k}-r_{-k}\right)=\frac{m}{k^{2}}\left\{2 \sin \frac{3 \pi k}{4}-\sin \frac{k \pi}{2}\right\}, \quad k=1,2,3, \cdots .
$$

Solving (3.2) for $p_{k}$ and $r_{-k}, k=1,2,3, \cdots$ we obtain

$$
\pi p_{k}=\frac{2}{k^{2}}\left\{\frac{2}{\pi}-\frac{4}{k \pi^{2}} \sin \frac{k \pi}{2}\right\}+\frac{m}{k^{2}}\left\{2 \sin \frac{3 \pi k}{4}-\sin \frac{k \pi}{2}\right\}
$$

and

$$
\pi r_{-k}=\frac{2}{k^{2}}\left\{\frac{2}{\pi}-\frac{4}{k \pi^{2}} \sin \frac{k \pi}{2}\right\}-\frac{m}{k^{2}}\left\{2 \sin \frac{3 \pi k}{4}-\sin \frac{k \pi}{2}\right\} .
$$

When $m<1 / \pi^{2}$, both $p_{k}$ and $r_{-k}$ are positive for all $k$, so that $\phi$ is the characteristic function of a probability distribution. 


\subsection{Sufficient conditions for Proposition (1.1).}

LEMMA 4.1. Let $X$ and $Y$ be independent random variables which have finite moments $E X^{2 n+1}$ and $E Y^{2 n+1}$, respectively, for some integer $n \geqq 0$. If $E X^{2 j+1}=E Y^{2 j+1}$ and $E(X+Y)^{2 j+1}=0$ for $j=0,1, \cdots, n$ then $E X^{2 j+1}=0$ for $j=0,1, \cdots, n$.

PRoof. The lemma is trivial for $n=0$. If it were true for $n-1$, then

$$
\begin{aligned}
0 & =E(X+Y)^{2 n+1}=\sum_{j=0}^{2 n+1}\left(\begin{array}{c}
2 n+1 \\
j
\end{array}\right) E X^{j} E Y^{2 n+1-j} \\
& =E X^{2 n+1}+E Y^{2 n+1}
\end{aligned}
$$

since either $j$ or $2 n+1-j$ is odd and less than $2 n+1$ for the remaining terms. Thus $E X^{2 n+1}=E Y^{2 n+1}=0$ and the lemma follows by induction.

THEOREM 4.1. If the characteristic function $\phi$ of a distribution function $F$ is either

(a) infinitely divisible or

(b) analytic

and if $F * F$ is symmetric, then $F$ is symmetric.

Proof. Assume (a) and note that if $\phi^{2}$ is real-valued, then $\phi$ has the form

$$
\begin{aligned}
\phi(t) & =u(t), & & t \in D, \\
& =i v(t), & & t \in \bar{D},
\end{aligned}
$$

where $D$ is a subset of the real line which contains the origin. If $\bar{D}$ is not void, the continuity of $\phi$ implies there exists a real $t_{0}$ such that $\phi\left(t_{0}\right)=0$. This contradicts assumption (a), since infinitely divisible characteristic functions have no real zeros $[1$, p. 80]. Thus $\bar{D}$ is void and $F$ is symmetric.

Now assume (b), and let $X, Y$ be independent random variables, each distributed as $F$. Since $\phi$ is analytic, moments of all orders exist and $\phi$ has a unique power series expansion $\phi(t)=\sum_{j=0}^{\infty}\left((i t)^{j} / j !\right) E X^{j}$. If the distribution $F * F$ of the sum $X+Y$ is symmetric, then the odd moments $E(X+Y)^{2 j+1}$ are zero for $j=0,1,2, \cdots$. Thus by Lemma 4.1, the odd moments $E X^{2 j+1}$ are also zero, $j=0,1,2, \cdots$. Hence $\phi(t)=\sum_{j=1}^{\infty}(-1)^{j}\left(t^{2 j} /(2 j !)\right) E X^{2 j}$ and $F$ is symmetric.

4.2. Discussion. The characteristic functions constructed in this paper are not differentiable at the origin so finite moments do not 
exist for their corresponding distribution functions. An open question is whether distributions having one or more finite moments can exhibit similar behaviour.

\section{BIBLIOGRAPHY}

1. Eugene Lukacs, Characteristic functions, Griffin's Statistical Monographs \& Courses, no. 5, Haf ner, New York, 1960. MR 23 \#A1392.

Michigan State University, East Lansing, Michigan 48823 